\title{
outra travessia
}

poesia: a voz

e a escuta 



\section{outra travessia}

Revista de Literatura $\mathrm{n}^{\circ} 15$

Ilha de Santa Catarina $1^{\circ}$ semestre de 2013

\section{poesia: a voz e a escuta}

Número organizado por

Susana Scramim

Luciana di Leone

Editores

Carlos Eduardo Schmidt Capela

Fernando Floriani Petry Jorge Wolff

Programa de Pós-Graduação em Literatura Universidade Federal de Santa Catarina 


\section{Ficha Técnica}

\section{Imagem Capa:}

Foto: Carlos Ríos sobre imagem de "Silence", de David Moreno.

\section{Diagramação:}

Cláudio José Girardi

\section{Catalogação}

ISSN: 0101-9570

eISSN: 2176-8552

\section{Editores do número 15:}

Susana Scramim/ Luciana di Leone

\section{Editores:}

Carlos Eduardo Schmidt Capela / Fernando Floriani Petry / Jorge Wolff

\section{Revisão:}

Diego Cervelin/ Marilha Naccari/ Ariele Louise Barichello Cunha

\section{Conselho Consultivo:}

Adriana Rodríguez Persico (Universidad de Buenos Aires, Argentina)

Ana Cecilia Olmos (Universidade de São Paulo, Brasil)

Ana Luiza Andrade (Universidade Federal de Santa Catarina, Brasil)

Ana Porrúa (Universidad Nacional de Mar del Plata, Argentina)

Antônio Carlos Santos (Universidade do Sul de Santa Catarina, Brasil)

Celia Pedrosa (Universidade Federal Fluminense, Brasil)

Daniel Link (Universidad de Buenos Aires/ Universidad Nacional de Tres de Febrero, Argentina)

Emanuele Coccia (École des Hautes Études en Sciences Sociales, França)

Ettore Finazzi-Agrò (Sapienza Università di Roma, Itália)

Fabián Ludueña Romandini (Universidad de Buenos Aires, Argentina)

Flora Süssekind (Fundação Casa de Rui Barbosa, Brasil)

Florencia Garramuño (Universidad de San Andrés, Argentina)

Francisco Foot-Hardman (Universidade Estadual de Campinas, Brasil)

Gabriela Nouzeilles (Princeton University, Estados Unidos)

Gema Areta (Universidad de Sevilla, Espanha)

Gonzalo Aguilar (Universidad de Buenos Aires, Argentina)

Ivia Alves (Universidade Federal da Bahia, Brasil)

Jair Tadeu da Fonseca (Universidade Federal de Santa Catarina, Brasil)

Liliana Reales (Universidade Federal de Santa Catarina, Brasil)

Luz Rodríguez Carranza (Universidad de Leiden, Holanda)

Marcos Siscar (Universidade Estadual de Campinas, Brasil)

Maria Augusta Fonseca (Universidade de São Paulo, Brasil)

Maria Esther Maciel (Universidade Federal de Minas Gerais, Brasil)

Raul Antelo (Universidade Federal de Santa Catarina, Brasil)

Rita Lenira Bittencourt (Universidade Federal do Rio Grande do Sul, Brasil)

Roberto Vecchi (Università di Bologna, Itália)

Sabrina Sedlmayer Pinto (Universidade Federal de Minas Gerais, Brasil)

Simone Pereira Schmidt (Universidade Federal de Santa Catarina, Brasil)

Wander Melo Miranda (Universidade Federal de Minas Gerais, Brasil)

Wladimir Garcia (Universidade Federal de Santa Catarina, Brasil) 


\section{Sumário}

Apresentação

7. Susana Scramim e Luciana di Leone

\section{A Voz, o outro}

Escuta e responsabilidade na relação com o outro em tradução

13. Mauricio Mendonça Cardozo

Lenguaje y voz. Pensamiento contemporáneo y experiencia poética

37. Gabriela Milone

De mistério e de letras: nenhum caminho por trás da linguagem?

57. Vinícius Nicastro Honesko

Escutando a voz de Meschonnic

75. Cristina Henrique da Costa

Escuchar, recolectar, configurar: inscripciones de la poesía popular

91. en Juan Alfonso Carrizo y Bernardo Canal Feijóo

Diego Bentivegna

Poema-partitura e poéticas vocais

113. Silvio Ferraz e Annita Costa Malufe

A terceira voz: por uma poética da tradução

133. Marcus Rogério Tavares Sampaio Salgado

Uma filosofia que seja o estremecer de um som: sobre o sentido em Jean-Luc Nancy 147. Paola Ghetti

\section{II $\grave{A}$ escuta}

Parte I de $\dot{A}$ escuta

159. Jean-Luc Nancy (trad. Carlos Eduardo Schmidt Capela e Vinícius Nicastro Honesko)

\section{Escutas poéticas}

La voz y el espacio: documentos artísticos de una filiación

175. Ana Porrúa

Impulso dramático. Entre Carlos de Oliveira e João Cabral de Melo Neto

189. Leonardo Gandolfi 
A performance da voz e a subjetividade na poesia contemporânea

205. Olliver Mariano Rosa e Goiandira Ortiz de Camargo

O Nunca Mais é a fera: ensaio sobre perda e predileção

227. Luciana Tiscoski

Contatos: Cruz e Sousa e Berna Reale 247. Maiara Knihs

Ana Cristina Cesar e Paulo Leminski trocam cartas

265. Joacy Ghizzi Neto

Restos audibles: entre la poesía y la historia en Punctum, de Martín Gambarotta 281. Mario Cámara

293.

Normas para Apresentação 